'DJ Carslake, 'G Davey Smith, ${ }^{2 D}$ Gunnell, ${ }^{3} \mathrm{TIL}$ Nilsen, $4 \mathrm{P}$ Romundstad. ${ }^{1} \mathrm{MRC}$ Centre for Causal Analyses in Translational Epidemiology, School of Social and Community Medicine, University of Bristol, Bristol, UK; ${ }^{2}$ School of Social and Community Medicine, University of Bristol, Bristol, UK; ${ }^{3}$ Department of Human Movement Science, Norwegian University of Science and Technology, Trondheim, Norway; ${ }^{4}$ Department of Public Health and General Practice, Norwegian University of Science and Technology, Trondheim, Norway

Background People with high body mass index (BMI) suffer elevated rates of mortality from a variety of causes, particularly from cardiovascular disease (CVD). Some studies have also found elevated mortality at low BMI, but it is unclear whether this represents a causal association. Potential confounding by existing ill-health is of particular concern. To avoid this problem, we used the BMI of an offspring as an instrument for the parent's BMI, as well as conducting conventional analyses of parental mortality against their own BMI.

Methods We extracted 33,011 mother-offspring and 28,142 fatheroffspring pairs from HUNT, a large prospective cohort study conducted in Nord-Trøndelag county, Norway. Participants' BMI was adjusted for age, sex, secular trends and smoking status. Associations of parental mortality rates with their own BMI were estimated as hazard ratios using Cox regression. Age was the time axis, and we adjusted for smoking, alcohol, exercise, education and employment. Additionally, adjusted causal hazard ratios between parental mortality and BMI were estimated using offspring BMI as an instrument for parental BMI and cubic splines were fitted to all associations to assess their linearity.

Results There were 9,271 maternal and 9,889 paternal deaths within the follow-up period of 1984-2009. The associations of all-cause, CVD and cancer mortality with parents' own BMI were substantially nonlinear, with elevated mortality at both extremes and minima at 21-25 $\mathrm{kg} \mathrm{m}^{-2}$. Hazard ratios for all-cause mortality per standard deviation $\left(4.1 \mathrm{~kg} \mathrm{~m}^{-2}\right)$ of own BMI were 1.04 (95\% CI: 1.02, 1.06) and 1.05 (1.02, 1.08 ) in mothers and fathers, respectively. In contrast, associations of mortality with offspring BMI were approximately linear and positive. Causal hazard ratios per standard deviation of BMI estimated from the instrumental variable analyses were $1.20(1.11,1.30)$ and 1.11 $(1.02,1.22)$ for all-cause mortality in mothers and fathers, respectively. For CVD they were $1.26(1.13,1.41)$ and $1.15(1.01,1.30)$, and for cancer they were $1.23(1.05,1.45)$ and $1.07(0.90,1.27)$.

Conclusion These results confirm the elevated mortality from all causes, CVD and cancer in subjects with high or low BMI. The use of an offspring's BMI as an instrument suggests that the elevated mortality at low BMI in this and other studies may be the result of confounding by existing ill-health, and that the causal relationship between BMI and mortality is positive and approximately linear.

\section{OP64 PARENTAL SUICIDE ATTEMPT AND OFFSPRING SELF- HARM AND SUICIDAL THOUGHTS: RESULTS FROM THE ALSPAC BIRTH COHORT}

doi:10.1136/jech-2012-201753.064

G Geulayov, C Metcalfe, DJ Gunnell. School of Social and Community Medicine, Bristol University, Bristol, UK

Background Exposure to parental self-harm has been linked to an increased risk of self-harm and suicidal thoughts in their offspring. Much of the available evidence is from population registers or clinical samples and relates to parental death by suicide; few studies have investigated associations of non-fatal suicide attempts in parents with community presenting non-fatal self-harm and suicidal thoughts in offspring. We studied the association of parental suicide attempt (SA) with offspring self-harm and suicidal thoughts using a large two-generational prospective cohort.

Methods The sample comprised 4,396 children, their mothers and 2,541 of the mothers' partners from the Avon Longitudinal Study of
Parents and Children (ALSPAC). Parents were asked to report on incident episodes of SA on 10 separate occasions from pregnancy until the children were 11 years old. Information on lifetime childhood self-harm, with and without suicidal intent, and suicidal thoughts, with and without suicidal plan, was collected through child-completed questionnaires at the age of 16-17 years.

Results Based on preliminary results, SA was reported by $1.5 \%$ of the mothers and $0.7 \%$ of the partners. Adjusting for sociodemographic factors and parental depression, maternal SA was associated with 3-fold increased risk of self-harm with suicidal intent in their children [Adjusted odds ratio (AOR) 3.0, 95\% confidence interval (CI) 1.4-6.1] but not with self-harm with no suicidal intent (AOR $0.8,95 \%$ CI 0.3-1.9). Children exposed to maternal SA were more likely than unexposed children to report both suicidal thoughts with and without suicidal plan (AOR 5.2, 95\% CI 2.3-11.6; AOR $2.2,95 \%$ CI 1.1-4.4, respectively). Partner SA was associated with 2.3-fold increased risk of self-harm with suicidal intent (95\% CI 0.5-10.6) and with an increased risk of suicidal thoughts with suicidal plan (AOR 3.5, 95\% CI 0.8-16.0) but these results are consistent with chance. There was no evidence of an association between partner suicide attempt with offspring self-harm with no suicidal intent (AOR 0.4, 95\% CI 0.1-3.5) or with offspring suicidal thoughts without a suicidal plan (AOR 0.6, 95\% CI 0.1-4.5).

Conclusion Parental SA in childhood increases the risk of selfharm with suicidal intent in offspring but is unrelated to risk of self-harm without suicide intent. Parental SA is associated with larger effect size on suicidal thoughts with a suicidal plan than without a plan. Findings provide limited evidence that maternal SA is a more potent risk factor than partner SA.

\section{HSR: Professional Behaviour and Health Care}

\section{OP65 PARENT UNDERSTANDING AND MANAGEMENT OF RTIS IN CHILDREN: IMPLICATIONS FOR HEALTH PRACTITIONER COMMUNICATION}

doi:10.1136/jech-2012-201753.065

CL Cabral, JP Horwood, AD Hay, JC Ingram. School of Social and Community Medicine, University of Bristol, Bristol, UK

Background Respiratory tract infections (RTIs) are among the commonest reason parents consult their GP, representing a significant burden on primary health care services. Many of the symptoms associated with RTIs are a cause of anxiety for parents, but they are often unsure when to consult. This study investigated parents' experiences of consulting for RTI in their child as part of a wider TARGET programme to improve the care of children with RTIs.

Methods Parents were recruited through 6 practices in areas of high, middle and low deprivation to capture a range of patient populations. Parents with a child aged between 3 months and 12 years who had consulted for acute RTI within the previous 3 months (excluding children with serious or chronic health problems) were invited to participate. Sampling ensured that parents with younger and older children and parents who were more or less frequent consulters were sampled. Semi-structured interviews explored parent's experience of consulting for RTIs in their children, information and advice needs; and their understanding of RTIs and treatment options. Interviews were audio-recorded, transcribed and imported into NVivo8 for coding. A thematic analysis was conducted using constant comparison techniques.

Results Thirty parents were interviewed and the sample captured a range of socio-economic backgrounds, both single and double parent families, with between 1 and 4 children. Consultation rates ranged from 1 to 24 times per year. New parents often had little knowledge of RTIs in children; felt uncertain about which 
symptoms might indicate a serious illness; and were surprised at how long a RTI could persist for and how frequently a child could get a RTI, particularly in the first years of life. This was true for parents from all socio-economic backgrounds and with different levels of education. When consulting, parents were often seeking a medical assessment and reassurance. However, parents felt that clinicians were often dismissive and had not properly evaluated the child, often leading to re-consultations. In addition, clinician explanations of diagnosis and treatment recommendations were not well understood by parents, and they remained unclear about how to manage an RTI and when to consult.

Conclusion Parents' poor knowledge and un-realistic expectations in relation to RTIs in children contribute to high rates of consultation. Despite awareness of parental perspectives, there remains a problem with parent-HP communication in relation to key information needed by parents to manage child's illness confidently and know when to consult in future.

\section{OP66 A PROFILE OF UNDIAGNOSED DIABETICS IN THE COMMUNITY: RESULTS FROM THE BOSTON AREA COMMUNITY HEALTH (BACH) PRE-DIABETES SURVEY}

doi:10.1136/jech-2012-201753.066

RS Piccolo, JB McKinlay, LD Marceau. Health Services and Disparities Research, New UK Research Institutes, Watertown, USA

Background Research suggests almost 30\% of the United States population has undiagnosed diabetes and that diabetics typically have the disease for 4 to 7 years before eventual diagnosis. Delay in initial diagnosis results in greater diabetes-related complications, poorer patient outcomes, and reduced quality of life. Most diabetes research is necessarily confined to diagnosed diabetes. Our objective was to identify risk factors for remaining undiagnosed.

Methods The Boston Area Community Health (BACH) Pre-Diabetes study is an ongoing community-based random-sample cohort study that has enrolled 2,974 participants to date. Participants were asked to fast for 8 hours prior to their interview. Fasting glucose (FG) and glycated hemoglobin (HbA1c) were collected during inhome interviews. Undiagnosed diabetes was defined as FG $>125$ $\mathrm{mg} / \mathrm{dL}$ or $\mathrm{HbA} 1 \mathrm{c} \geq 6.5 \%$. Risk factors were organized into four logical groupings: 1) socio-demographics, 2) lifestyle/behavioral, 3) utilization/access to healthcare, and 4) health status/comorbidities. Logistic regression was used to estimate the odds ratio (OR) for diagnosed vs. undiagnosed diabetes.

Results The prevalence of diabetes in the BACH study was $27.8 \%$ $(n=827) .21 .2 \%$ of diabetes cases were undiagnosed. The data indicate that healthcare utilization and the presence of co-morbid conditions had a large impact on diabetes diagnosis. Participants who visited a health care provider 5 or more times in the past year were $90 \%$ more likely to be diagnosed $(\mathrm{OR}=1.9, \mathrm{p}<0.001)$ than participants who had 5 or fewer visits. Participants with a history of high cholesterol were more than twice as likely to be diagnosed $(\mathrm{OR}=2.7$, $p<0.001)$. High blood pressure or a history of heart disease also increased the likelihood a participant was diagnosed. Participants with a documented family history of diabetes were more likely to be diagnosed $(\mathrm{OR}=2.9, \mathrm{p}<0.001)$. Finally, English-speaking participants were more than twice as likely to be diagnosed $(\mathrm{OR}=2.6, \mathrm{p}=0.01)$ than their Spanish-speaking counterparts.

Conclusion Undiagnosed diabetes is highly prevalent problem in the United States that leads to poorer patient outcomes and significant health-care costs. These results indicate that access to care, health care utilization and the presence of co-morbid conditions have an important impact on diabetes diagnosis. Individuals who do not have the traditional risk factors for diabetes (i.e. family history) have a greater risk of remaining undiagnosed. These findings suggest that improving access to care in the United States may greatly increase the likelihood of diagnosing previously undiagnosed diabetes.

\section{OP67 DO DOCTORS CONTRIBUTE TO THE SOCIAL PATTERNING OF DISEASE? CONTRASTING FINDINGS FROM AN EPIDEMIOLOGIC SURVEY AND A DECISION MAKING EXPERIMENT}

doi:10.1136/jech-2012-201753.067

J.B. McKinlay. Health Services and Disparities Research, New England Research Institutes, Watertown, USA

Background That type 2 diabetes mellitus (T2DM) varies significantly by race and ethnicity is a widely accepted fact. It is often invoked as a base rate (a priori probability) during the process of clinical reasoning. Epidemiological studies repeatedly show undiagnosed T2DM varies more by socioeconomic status (SES), than by race/ethnicity. This study seeks to understand the discrepancy between the true prevalence of undiagnosed T2DM by SES and its continually reported prevalence by race/ethnicity.

Methods Data from two different but complementary studies are employed: a) a large Boston Area Community Health (BACH) survey; and b) a factorial experiment conducted with primary care doctors to examine variations in clinical decision making. The $\mathrm{BACH}$ epidemiologic survey $(\mathrm{n}=5502)$ employed a stratified, multi-stage cluster sample design and used multivariable techniques including logistic regression. The factorial experiment concerning decision making employed clinically authentic videotaped scenarios presented to primary care doctors $(\mathrm{n}=192)$, and used ANCOVA analyses.

Results Results from the epidemiologic survey show that both undiagnosed signs and symptoms and diagnosed T2DM vary similarly by socio-economic status (SES). This finding is independently corroborated by National Health and Nutrition Examination Survey (NHANES) data for diagnosed T2DM. Complementary data from the clinical decision making experiment show the diagnosis of T2DM varies significantly by a patients' race/ethnicity, controlling for SES, age and gender in the design. While undiagnosed signs and symptoms of T2DM in the community vary significantly by SES, rather than race/ethnicity, following diagnosis by primary care doctors they vary more by race/ethnicity, rather than by SES.

Conclusion Race/ethnicity and SES in the US are almost totally confounded, such that measuring one is essentially also measuring the other. Consequently, doctors generally get the social patterning of T2DM right, but for entirely the wrong reason. Continued patterning of T2DM by race/ethnicity motivates the search for genetic and biophysiologic explanations and distracts attention from the more important and potentially modifiable contribution of SES circumstances to the prevalence of T2DM.

\section{OP68 THE CONTRIBUTION OF DOCTORS' DECISIONS TO HEALTHCARE INEQUALITIES: TOWARDS FOURTH GENERATION STUDIES}

doi:10.1136/jech-2012-201753.068

LD Marceau, JB McKinlay, RS Piccolo. Health Services and Disparities Research, New UK Research Institutes, Watertown, USA

Background Healthcare variations are a worrisome and well-documented problem. Such variations occur at different levels: between health care systems, geographic areas, organizational settings, patients, and doctors. Research is increasingly focused on the contribution of doctors' decision making to the generation or amplification of healthcare disparities. Clinical decision making studies continue to evolve through generations of work that focus on different types of influence: First generation studies identified patient-level attributes (e.g., gender, age, race/ethnicity, SES, health insurance status); Second generation studies on variations associated with physician characteristics (e.g., age, gender and education, specialty and type of remuneration); Third generation studies on health system and 